\title{
Strain Rates in the Sichuan-Yunnan Region Based upon the Total Least Squares Heterogeneous Strain Model from GPS Data
}

\author{
Caijun $\mathrm{Xu}^{1,2, *}$, Leyang Wang ${ }^{1}$, Yangmao $\mathrm{Wen}^{1,2}$, and Jianjun Wang ${ }^{1}$ \\ ${ }^{1}$ School of Geodesy and Geomatics, Wuhan University, Wuhan, China \\ ${ }^{2}$ Key Laboratory of Geo-Space Environment and Geodesy, Ministry of Education, Wuhan University, Wuhan, China
}

Received 20 January 2009, accepted 26 July 2010

\begin{abstract}
We present crustal strain and deformation models for the Sichuan-Yunnan region based on high-precision GPS measurements from 1998 - 2004 using the total least squares method (TLSM). Coordinate errors as well as GPS velocity errors recorded at GPS stations are considered, but only the latter errors are considered using the conventional least squares method (LSM). In addition, the spatial pattern of a given strain field is also likely to be heterogeneous. We investigate two models with a spatially variable strain, the least squares heterogeneous strain model (LS-HSM) and the total least squares heterogeneous strain model (TLS-HSM). Our result shows that estimated strain field parameters are more precise using the TLS-HSM than those by LS-HSM because the fitting to the data is improved, hence the TLS-HSM is preferred. The principal dilation strain rate, principal contraction strain rate, maximum shearing strain rate and surface dilation rate estimated by TLS-HSM in the northwestern Sichuan-Yunnan sub-block are 13.2526 $\pm 1.2624,-10.8001 \pm 2.9826,24.0527 \pm 3.2381$, and $2.4525 \pm$ $3.2393 \times 10^{-9} \mathrm{yr}^{-1}$ (with a confidence probability of 95\%), respectively, while those in the southeastern Sichuan-Yunnan subblock are $18.8651 \pm 1.8353,-12.0875 \pm 1.3926,30.9525 \pm 2.2971$ and $6.7776 \pm 2.3105 \times 10^{-9} \mathrm{yr}^{-1}$ (and exhibiting similar probabilities), respectively. The results indicate that the sub-blocks play a key role in continental tectonic deformation in the Sichuan-Yunnan region, and that small errors in site coordinates can have a significant impact on strain estimates, especially where sites are close together.
\end{abstract}

Key words: Total Least Squares (TLS), Strain rate, GPS, Uniform strain model (USM), Heterogeneous strain model (HSM)

Citation: Xu, C., L. Wang, Y. Wen, and J. Wang, 2011: Strain rates in the Sichuan-Yunnan region based upon the total least squares heterogeneous strain model from GPS data. Terr. Atmos. Ocean. Sci., 22, 133-147, doi: 10.3319/TAO.2010.07.26.02(TibXS)

\section{INTRODUCTION}

The Sichuan-Yunnan region is located in the southeast borderland of the Tibetan Plateau and is characterized by a complex geological structure (Fig. 1). The structure is adjacent to the stable south China block to the east, and spans most of Sichuan and Yunnan provinces. There have been many, widely-distributed, shallow hypocenter and great-magnitude earthquakes in the area. For example, the 12 May 2008 Wenchuan $M_{w} 7.9$ earthquake occurred along the Longmenshan fault zone. Determination of crustal deformation and its stress and strain characteristics using geodetic data is a very effective method for investigating the regularity of crustal movement, determining earthquake

\footnotetext{
* Corresponding author

E-mail: cjxu@sgg.whu.edu.cn
}

potential and provides direct evidence via quantitative analysis of characteristics within the geophysical meaning of crustal movement. Recently, there have been many studies using GPS observation data to obtain crustal horizontal displacements and strain characteristics about the SichuanYunnan region (e.g., Lü et al. 2003; Shen et al. 2005; Gan et al. 2007; Xu et al. 2007; Wang et al. 2008). Although the crustal deformation models employed in these studies were not exactly the same, all used the least squares method (LSM) wherein only errors of GPS velocities were considered and not the coordinates of GPS stations. Just as importantly GPS site coordinates reveal actual errors of less than centimeters. A typical 3-D weighted root-meansquare (WRMS) misfit after transformation is $4-6 \mathrm{~mm}$ for recent GPS sites solutions, while the post transformation 3-D WRMS residuals were usually $\sim 15 \mathrm{~mm}$ for 1993 and 


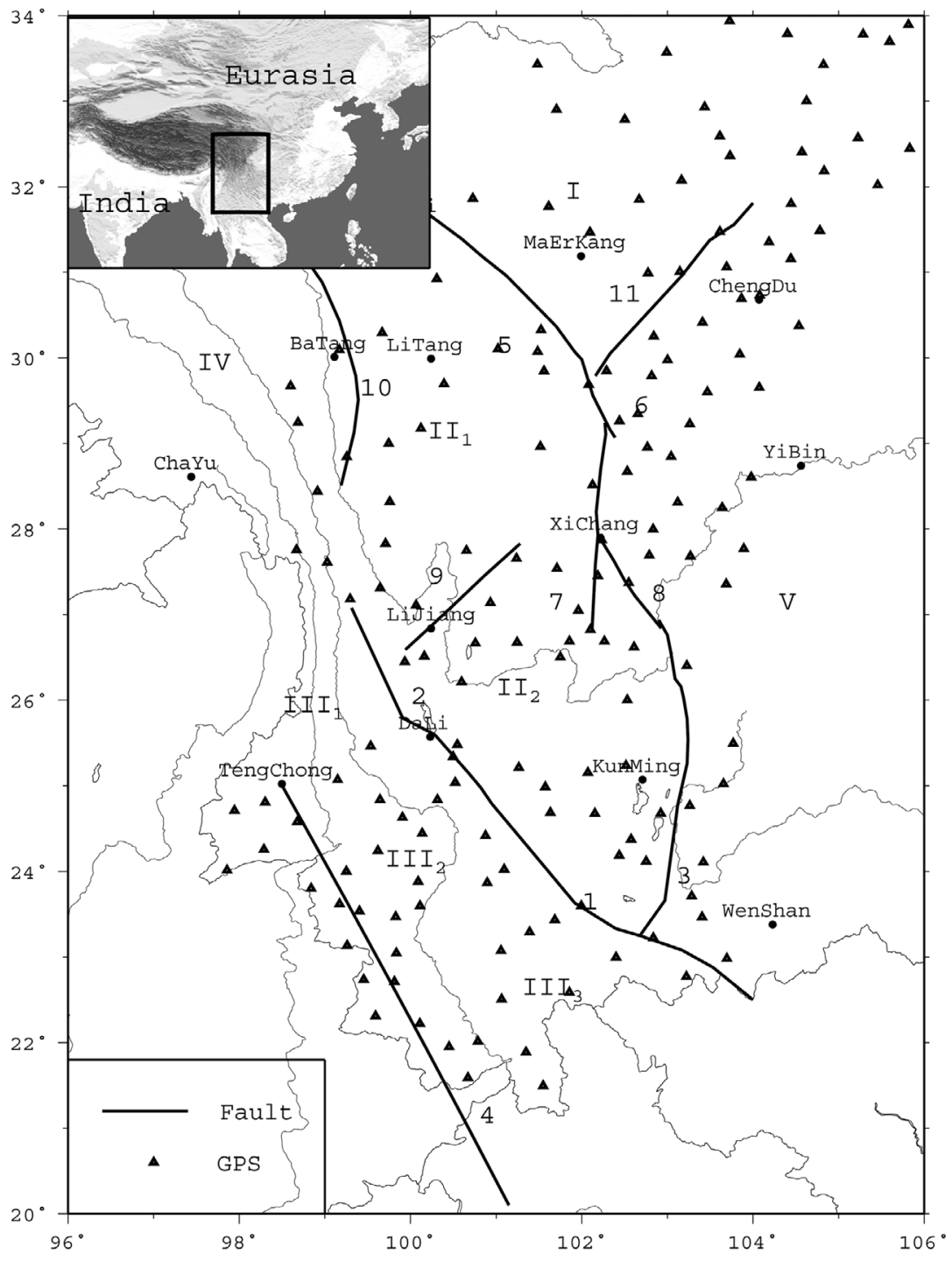

Fig. 1. Tectonic map of the Sichuan-Yunnan Region from Xu and Wen (2007). The studied region is depicted in the inset map. I: the Maerkang block; II1: the north-western Sichuan sub-block; II2: the central Yunnan sub-block; III1: the Baoshan sub-block; III2: the Jinggu sub-block; III3: the Mengla sub-block; IV: the Qiangtang block; V: the southern China block. 1: the southern segment of Red River Fault; 2: the northern segment of Red River Fault; 3: the Xiaojiang Fault; 4: the Tengchong-Jinghong Fault; 5: the northern segment of Xianshuihe Fault; 6: the southern segment of Xianshuihe Fault; 7: the Anninghe Fault; 8: the Zemuhe Fault; 9: the Lijiang Fault; 10: the Jinshajiang Fault; 11: the Longmenshan Fault. The lines denote the trace of the fault segments. The triangles denote the GPS survey stations.

20 $\mathrm{mm}$ for 1992 (Freymueller et al. 2008). Segall and Davis (1997) thought that individual GPS measurements across regional scales had accuracies of $2-5 \mathrm{~mm}$ for horizontal components and were a factor of 3 worse for vertical components; Dietrich et al. (2001) obtained a combined solution with an accuracy of $1 \mathrm{~cm}$ for the horizontal plane and $2 \mathrm{~cm}$ for the vertical coordinate components within ITRF96; Larson et al. (2007) improved the standard deviations to 3.0 and $2.6 \mathrm{~mm}$ for the north and east components of the high-rate GPS, respectively. Although the errors of GPS site coordinates are not very large, they can have a real impact on the estimations of strain rate. Sometimes, the distribution of GPS sites is heterogeneous, and two GPS sites may be too close to each other. If so, the rows of the coefficient matrix may be related, and small errors in the coefficient matrix may cause large errors in the estimation. Because the coefficient matrix of the strain rate model is comprised of coordinates, we should consider possible errors in coordinate assignment in the coefficient matrix. These errors can be taken into account using the estimator of the total least squares (TLS), which has been widely investigated (e.g., Golub and van Loan 1980; van Huffel and Vandewalle 1991; Schaffrin 2006; Schaffrin and Wieser 2008; Markovsky and van Huffel 2007; Zhou and Lu 2008; Wang et al. 2010).

In this study, high-precision GPS velocities data and a linear heterogeneous strain and rotation model based on 
total least squares heterogeneous strain model (TLS-HSM) are used to investigate the strain features and deformation patterns in the Sichuan-Yunnan region.

\section{INVERSION MODELS OF BLOCK STRAIN PA- RAMETERS}

\subsection{Uniform Strain Model (USM)}

If the observed motion of a small array is approximated by an average strain rate within the array plus a rigid movement of the array as a whole, the approximation in two dimensions in spherical coordinates would be as follows (Savage et al. 2001; Xu and Wen 2007).

$$
\left\{\begin{aligned}
\bar{v}_{n}= & \bar{V}_{n}-\sin \varphi_{0} \Delta \lambda \bar{V}_{e}+r_{0} \Delta \varphi \dot{\varepsilon}_{n} \\
& +\frac{r_{0} \cos \varphi_{0} \Delta \lambda \dot{\gamma}_{e n}}{2}-r_{0} \cos \varphi_{0} \Delta \lambda \omega \\
\bar{v}_{e}= & \bar{V}_{e}-\sin \varphi_{0} \Delta \lambda \bar{V}_{n}+r_{0} \cos \varphi_{0} \Delta \lambda \dot{\varepsilon}_{e} \\
& +\frac{r_{0} \Delta \varphi \dot{\gamma}_{e n}}{2}+r_{0} \Delta \varphi \omega
\end{aligned}\right.
$$

where $\left(\bar{v}_{n}, \bar{v}_{e}\right)$ are the velocities at observed GPS stations; $\left(\bar{V}_{n}, \bar{V}_{e}\right)$ are the velocities at the centroid of the array; $\Delta \lambda$ and $\Delta \varphi$ are the angular distances from the centroid; $\dot{\mathcal{E}}_{e}, \dot{\mathcal{E}}_{n}$ and $\dot{\gamma}_{e n}$ are the tensor strain rate components, and $\omega$ is the rotation rate about the local vertical (positive direction is clockwise as viewed from above). If the unknowns $\bar{V}_{n}, \bar{V}_{e}$, $\dot{\varepsilon}_{e}, \dot{\varepsilon}_{n}, \dot{\gamma}_{e n}$ and $\omega$ are inverted using least squares, this model is termed the least squares uniform strain model (LSUSM).

\subsection{Heterogeneous Strain Model (HSM)}

Block motion is regarded as a linear strain and rotation rather than a rigid motion in our study. In a block, using a Gaussian projection, the latitude and longitude coordinates of GPS stations are transferred to Gaussian plane rectangular coordinates. The coordinate transformation is computed for each block, individually, using the center longitude of each block. North is the upward positive direction of the $x$ axis; the east is the positive, horizontal direction of the $y$ axis. Here, $(x, y)$ denotes the Gaussian plane coordinates of a station. The station velocity is divided into $v_{n}$ (along a northerly direction) and $v_{e}$ (along an easterly direction).

From the elastic block motion equations, the strain rate can be written following $\mathrm{Li}$ et al. 2007 and Hao et al. 2009 as

$$
\left\{\begin{array}{l}
d v_{n}=\varepsilon_{n} d x+\varepsilon_{e n} d y+\omega_{s} d y \\
d v_{e}=\varepsilon_{e} d y+\varepsilon_{e n} d x-\omega_{s} d x
\end{array}\right.
$$

where $\left(v_{n}, v_{e}\right)$ are the north and east components of velocity; $(x, y)$ represent the coordinates of the station in a Gaussian plane; $\varepsilon_{n}, \varepsilon_{e}$ and $\varepsilon_{e n}$ denote a S-N, E-W direction strain rate, respectively and between an E-W and S-N direction shear strain rate tensor. Accordingly, $\omega_{s}$ represents the rotation rate which is negative when rotation is clockwise as viewed from above. The contractive strain rate is negative and the dilatation strain rate is positive.

In the heterogeneous model, if we assume the strain rate tensor $\varepsilon_{n}, \varepsilon_{e}$ and $\varepsilon_{e n}$, and rotation rate $\omega_{s}$ are a linear function of the coordinates $(x, y)$ following Li et al. (2007) and Hao et al. (2009), then

$\left\{\begin{array}{l}\mathcal{E}_{n}=A_{0}+A_{1} x+A_{2} y \\ \mathcal{E}_{e}=B_{0}+B_{1} x+B_{2} y \\ \mathcal{E}_{e n}=C_{0}+C_{1} x+C_{2} y \\ \omega_{s}=D_{0}+D_{1} x+D_{2} y\end{array}\right.$

where $A_{0}, A_{1}, A_{2}, B_{0}, B_{1}, B_{2}, C_{0}, C_{1}, C_{2}, D_{0}, D_{1}$, and $D_{2}$, are undetermined coefficients.

Substituting Eq. (3) into Eq. (2), and integrating both sides of the formula, we get

$$
\left[v_{n}\right]=\left[\begin{array}{cc}
1 & 0 \\
0 & 1 \\
x & 0 \\
\frac{1}{2} x^{2} & 0 \\
x y & -\frac{1}{2} x^{2} \\
0 & y \\
-\frac{1}{2} y^{2} & x y \\
0 & \frac{1}{2} y^{2} \\
y & x \\
0 & x^{2} \\
y^{2} & 0 \\
y & -x
\end{array}\right]^{T}
$$

where $\Omega=\left[\begin{array}{llllllllllll}v_{n 0} & v_{e 0} & A_{0} & A_{1} & A_{2} & B_{0} & B_{1} & B_{2} & C_{0} & C_{1} & C_{2} & D_{0}\end{array}\right]^{\mathrm{T}}$, $\Omega \in R^{12 \times 1} ; D_{1}=A_{2}-C_{1} ; D_{2}=C_{2}-B_{1}$. The model is named as the heterogeneous strain model (HSM), and because the strain tensor is coordinate-dependent as shown by Eq. (3), and it can describe strain features that vary in the different parts of the block. When the Eq. (4) is inverted using estimator of least squares, this model is termed least squares heterogeneous strain model (LS-HSM). 
If the number of GPS stations is $m$, then

$$
\left[\begin{array}{c}
v_{n_{1}} \\
v_{e_{1}} \\
\vdots \\
v_{n_{m}} \\
v_{e_{m}}
\end{array}\right]=\left[\begin{array}{ccccc}
1 & 0 & \cdots & 1 & 0 \\
0 & 1 & \cdots & 0 & 1 \\
x_{1} & 0 & \cdots & x_{m} & 0 \\
\frac{1}{2} x_{1}^{2} & 0 & \cdots & \frac{1}{2} x_{m}^{2} & 0 \\
x_{1} y_{1} & -\frac{1}{2} x_{1}^{2} & \cdots & x_{m} y_{m} & -\frac{1}{2} x_{m}^{2} \\
0 & y_{1} & \cdots & 0 & y_{m} \\
-\frac{1}{2} y_{1}^{2} & x_{1} y_{1} & \cdots & -\frac{1}{2} y_{m}^{2} & x_{m} y_{m} \\
0 & \frac{1}{2} y_{1}^{2} & \cdots & 0 & \frac{1}{2} y_{m}^{2} \\
y_{1} & x_{1} & \cdots & y_{m} & x_{m} \\
0 & x_{1}^{2} & \cdots & 0 & x_{m}^{2} \\
y_{1}^{2} & 0 & \cdots & y_{m}^{2} & 0 \\
y_{1} & -x_{1} & \cdots & y_{m} & -x_{m}
\end{array}\right]^{T} \Omega
$$

Let $L=\left[\begin{array}{llll}v_{n_{1}} & v_{e 1} \cdots v_{n_{m}} & v_{e_{m}}\end{array}\right]^{T}, L \in R^{2 m \times 1}$;

$$
B_{1}=\left[\begin{array}{lllll}
1 & 0 & \cdots & 1 & 0 \\
0 & 1 & \cdots & 0 & 1
\end{array}\right]^{T}, B_{1} \in R^{2 m \times 2} ;
$$$$
B_{2}=\left[\begin{array}{ccccc}
x_{1} & 0 & \cdots & x_{m} & 0 \\
\frac{1}{2} x_{1}^{2} & 0 & \cdots & \frac{1}{2} x_{m}^{2} & 0 \\
x_{1} y_{1} & -\frac{1}{2} x_{1}^{2} & \cdots & x_{m} y_{m} & -\frac{1}{2} x_{m}^{2} \\
0 & y_{1} & \cdots & 0 & y_{m} \\
-\frac{1}{2} y_{1}^{2} & x_{1} y_{1} & \cdots & -\frac{1}{2} y_{m}^{2} & x_{m} y_{m} \\
0 & \frac{1}{2} y_{1}^{2} & \cdots & 0 & \frac{1}{2} y_{m}^{2} \\
y_{1} & x_{1} & \cdots & y_{m} & x_{m} \\
0 & x_{1}^{2} & \cdots & 0 & x_{m}^{2} \\
y_{1}^{2} & 0 & \cdots & y_{m}^{2} & 0 \\
y_{1} & -x_{1} & \cdots & y_{m} & -x_{m}
\end{array}\right]^{T}, B_{2} \in R^{2 m \times 10} ;
$$

$B=\left[B_{1}, B_{2}\right], B \in R^{2 m \times 12} ;$

$\Omega=\left[\Omega_{1}^{T}, \Omega_{2}^{T}\right]^{T}, \Omega \in R^{12 \times 1} ;$

$\Omega_{1}=\left[\begin{array}{ll}v_{n 0} & v_{e 0}\end{array}\right]^{T}, \Omega_{1} \in R^{2 \times 1}$;

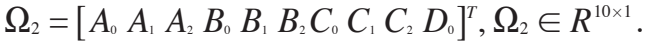

Then, the Eq. (5) can be written as
Now if both errors of the coordinates of the GPS stations and the errors of displacements (or velocities) recorded at GPS stations are considered, namely, there are errors emanating from coefficient matrix $B_{2}$ and observation vector $L$, estimator of TLS can be employed. In this case, the Eq. (6) can be written as

$L+e=B_{1} \Omega_{1}+\left(B_{2}+E_{B_{2}}\right) \Omega_{2}$

where $L$ is the observation vector affected by the random error vector $e$; and, the coefficient matrix $B_{2}$ is affected by the random error matrix $E_{B_{2}}$ :

$\left[\begin{array}{c}e \\ e_{B_{2}}\end{array}\right]=\left[\begin{array}{c}e \\ \operatorname{vec}\left(E_{B_{2}}\right)\end{array}\right] \sim\left\{\left[\begin{array}{l}0 \\ 0\end{array}\right], \sigma_{0}^{2} Q\left(e, e_{B_{2}}\right)\right\}$

where $\operatorname{vec}\left(E_{B_{2}}\right)$ is a column vector of $E_{B_{2}}$; "vec" denotes the operator that stacks one row of a matrix rearwards of the previous one, and then transposes it to obtain a column vector; $\sigma_{0}^{2}$ is an (unknown) variance component, $Q\left(e, e_{B_{2}}\right)$ is the cofactor matrix of $e$ and $e_{B_{2}}$. Backtracking slightly, from Eq. (7), we can obtain

$\bar{e}=e-E_{B_{2}} \Omega_{2}=B_{1} \Omega_{1}+B_{2} \Omega_{2}-L=B \Omega-L$

In the calculation, we first estimate the strain rate parameters by least squares method without considering all the errors in the coefficient matrix. Then we use the LS result as the initial value for the TLS inversion; namely, $\Omega_{2}^{0}$ becomes the approximate value of $\Omega_{2}$ based upon the least squares estimation from LS-HSM. Because both the TLS correction $\hat{\Omega}_{2}$ and $\hat{E}_{B_{2}}$ of the coefficient matrix $B_{2}$ are very small, we can ignore the quadratic term $E_{B_{2}} \hat{\Omega}_{2}$, and then postulate $\bar{e}=e-E_{B_{2}} \Omega_{2} \approx e-E_{B_{2}} \Omega_{2}^{0}$ based upon

$\bar{e}=\left[\begin{array}{c}e_{{ }_{11}} \\ e_{e_{1}} \\ \vdots \\ e_{n_{m}} \\ e_{e_{m}}\end{array}\right]-\left[\begin{array}{ccccc}e_{1,1} & 0 & \cdots & e_{2 m-1,1} & 0 \\ e_{1,2} & 0 & \cdots & e_{2 m-1,2} & 0 \\ e_{1,3} & e_{2,3} & \cdots & e_{2 m-1,3} & e_{2 m, 3} \\ 0 & e_{2,4} & \cdots & 0 & e_{2 m, 4} \\ e_{1,5} & e_{2,5} & \cdots & e_{2 m-1,5} & e_{2 m, 5} \\ 0 & e_{2,6} & \cdots & 0 & e_{2 m, 6} \\ e_{1,7} & e_{2,7} & \cdots & e_{2 m-1,7} & e_{2 m, 7} \\ 0 & e_{2,8} & \cdots & 0 & e_{2 m, 8} \\ e_{1,9} & 0 & \cdots & e_{2 m-1,9} & 0 \\ e_{1,10} & e_{2,10} & \cdots & e_{2 m-1,10} & e_{2 m, 10}\end{array}\right]_{2}^{T}=K \cdot \operatorname{vec}(G)$

where, $M=\left[\begin{array}{lllllllllll}1 & -A_{0}^{0} & -A_{1}^{0} & -A_{2}^{0} & -B_{0}^{0} & -B_{1}^{0} & -B_{2}^{0} & -C_{0}^{0} & -C_{1}^{0}\end{array}\right.$ 
$\left.-C_{2}^{0} \quad-D_{0}^{0}\right], M \in R^{1 \times 11} ; K=\left[\begin{array}{ccc}M & & \\ & \ddots & \\ & & M\end{array}\right], K \in R^{2 m \times 22 m} ; G \in R^{2 m \times 11}$, $\operatorname{vec}(G) \in R^{22 m \times 1}, \operatorname{vec}(G)$ is a column vector of $G$,

$G=\left[\begin{array}{ccccc}e_{n_{1}} & e_{e_{1}} & \cdots & e_{n_{m}} & e_{e_{m}} \\ e_{1,1} & 0 & \cdots & e_{2 m-1,1} & 0 \\ e_{1,2} & 0 & \cdots & e_{2 m-1,2} & 0 \\ e_{1,3} & e_{2,3} & \cdots & e_{2 m-1,3} & e_{2 m, 3} \\ 0 & e_{2,4} & \cdots & 0 & e_{2 m, 4} \\ e_{1,5} & e_{2,5} & \cdots & e_{2 m-1,5} & e_{2 m, 5} \\ 0 & e_{2,6} & \cdots & 0 & e_{2 m, 6} \\ e_{1,7} & e_{2,7} & \cdots & e_{2 m-1,7} & e_{2 m, 7} \\ 0 & e_{2,8} & \cdots & 0 & e_{2 m, 8} \\ e_{1,9} & 0 & \cdots & e_{2 m-1,9} & 0 \\ e_{1,10} & e_{2,10} & \cdots & e_{2 m-1,10} & e_{2 m, 10}\end{array}\right]^{T}$.

If we obtain a cofactor matrix $Q_{\operatorname{vec}(G)}\left[Q_{\operatorname{vec}(G)} \in R^{22 m \times 22 m}\right]$, according to the cofactor propagation law and Eq. (10), we can furthermore obtain

$Q_{\bar{e}}=K Q_{\operatorname{vec}(G)} K^{T}$

where $Q_{\operatorname{vec}(G)}=Q\left(e, e_{B_{2}}\right)$. According to the total least squares criterion, which can consider the errors of observation and coefficient matrix at the same time.

$\bar{e}^{T} P_{\bar{e}} \bar{e}=\min$

Following the traditional Lagrange approach and partial derivative, we can obtain

$$
\begin{aligned}
\frac{\partial \hat{\bar{e}}^{T} P_{\overline{\bar{e}}} \hat{\bar{e}}}{\partial \hat{\Omega}} & =2 \hat{\bar{e}}^{T} Q_{\bar{e}}^{-1} B+2 \hat{\bar{e}}^{T} \hat{\bar{e}} \frac{\partial Q_{\bar{e}}^{-1}}{\partial \hat{\Omega}} \\
& =2 \hat{\bar{e}}^{T} Q_{\bar{e}}^{-1} B+2 \hat{\bar{e}}^{T} \hat{\bar{e}} \frac{\partial\left[K Q_{\operatorname{vec}(G)} K^{T}\right]^{-1}}{\partial \hat{\Omega}} \\
& =0
\end{aligned}
$$

Because $\hat{\bar{e}}^{T} \hat{e}$ is very small and can be ignored, in this condition, from Eqs. (9) and (11) we can obtain

$\hat{\Omega}=\left(B^{T} Q_{\bar{\iota}}^{-1} B\right)^{-1} B^{T} Q_{\bar{\iota}}^{-1} L$

The symbol $\hat{\Omega}$ represents the parameters estimated from the TLS method with regard to the consideration of errors regarding coordinate displacement observations (GPS velocities) and the coefficient matrix. According to Eq. (3), we can determine all strain rate parameters. The model of Eq. (5) using the total least squares method (TLSM) is termed the total least squares heterogeneous strain model (TLS-HSM). The unit weight variance estimation formula is

$\hat{\sigma}_{0}^{2}=\frac{\hat{\bar{e}}^{T} Q_{\bar{e}}^{-1} \hat{\bar{e}}}{2 m-12}$

where $\hat{\bar{e}}=B \hat{\Omega}-L=\left[B\left(B^{T} Q_{\bar{e}}^{-1} B\right)^{-1} B^{T} Q_{\bar{e}}^{-1}-I_{2 m}\right] L$. The cofactor matrix is

$Q_{\hat{\alpha} \hat{\Omega}}=\left(B^{T} Q_{\bar{e}}^{-1} B\right)^{-1} B^{T} Q_{\bar{e}}^{-1} Q_{e} Q_{\bar{e}}^{-1} B\left(B^{T} Q_{\bar{e}}^{-1} B\right)^{-1}$

After obtaining $\varepsilon_{n}, \varepsilon_{e}$ and $\varepsilon_{e n}$, and rotation rate $\omega_{s}$, we can gain other parameters, such as the maximum shear strain rate $\dot{\gamma}_{\max }$, the area strain rate $\dot{\Delta}$, the contraction strain rate $\dot{\varepsilon}_{1}$ and the dilatation strain rate $\dot{\varepsilon}_{2}$, and so on (Xu et al. 2006).

\section{AN INVERSION OF BLOCK STRAIN RATES IN THE SICHUAN-YUNNAN REGION}

\subsection{Geological Setting}

Based upon the concept of "active blocks" and spatial distribution of recorded earthquakes with surface ruptures as well as major and subordinate active faults, the SichuanYunnan region can be divided into four first-order blocks (Xu et al. 2003; Xu and Wen 2007). They are the Maerkang block (I), the Sichuan-Yunnan rhombic block (II), the Baoshan-Pu'er block (III), and the Mizhina-Ximeng block (IV). Cut by sub-ordinate NE-trending active faults, the Sichuan-Yunnan rhombic block (II) can be further divided into two sub-blocks: the north-western Sichuan sub-block (II1) and central Yunnan sub-block (II2). The BaoshanPu'er block (III) can be further divided into three subblocks: the Baoshan sub-block (III1), the Jinggu sub-block (III2), and the Mengla sub-block (III3). Among all faults on the boundaries of those sub-blocks, the XianshuiheXiaojiang fault system is the most active (Wang et al. 1998). The entire fault system is about $1200 \mathrm{~km}$ long and a few hundred meters wide in most places, except across the mid and southern parts of the Xiaojiang fault where it splits into multiple branches spanning a range of $20-30 \mathrm{~km}$ (Shen et al. 2005).

Crustal deformation due to India-Eurasian collision is intense in this region, and many large earthquakes have occurred here. $14 \mathrm{M}>7.0$ earthquakes were recorded historically since 814 , including an event of $\mathrm{M}=8.0$ in 1833 (Zhang et al. 2001), with a maximum focal depth of $\sim 20 \mathrm{~km}$ (Tang et al. 1993). The 12 May 2008 Wenchuan earthquake 
occurred along the Longmen Shan fault zone, which is predominantly a convergent zone with a dextral component, separating the Sichuan basin from the eastern margin of the Tibetan plateau (Burchfiel et al. 2008). The seismic moment released during the Wenchuan earthquake mainshock was measured at $7.6 \times 10^{20} \mathrm{Nm}$ by the United States Geological Survey, corresponding to $\mathrm{M}_{\mathrm{w}} 7.9$ (USGS 2008).

\subsection{GPS Velocities}

GPS data for the Sichuan-Yunnan region used in our paper are from Shen et al. (2005), and were previously used by Xu and Wen (2007) as well. The GPS data include 249 GPS velocities and their variance-covariance matrix, and are mainly from the Crustal Motion Observation Network of
China (CMONOC) project. These data include data from a nationwide fiducial network of 25 continuous sites observed from July 1998 to October 2004, and 56 campaign survey sites with yearly occupations from 1998 to 2004, and include more than 200 regional campaign survey stations occupied in 1999, 2001 and 2004 (Shen et al. 2005). All survey mode sites were observed continuously for at least 4 days during each session. The velocity solution is referred to the Eurasian plate (Fig. 2), with the uncertainties of $2 \mathrm{~mm} \mathrm{yr}^{-1}$ for their east and north.

\subsection{Inversion Results}

We first estimate the strain rate parameters of HSM by the least squares method without considering all the errors

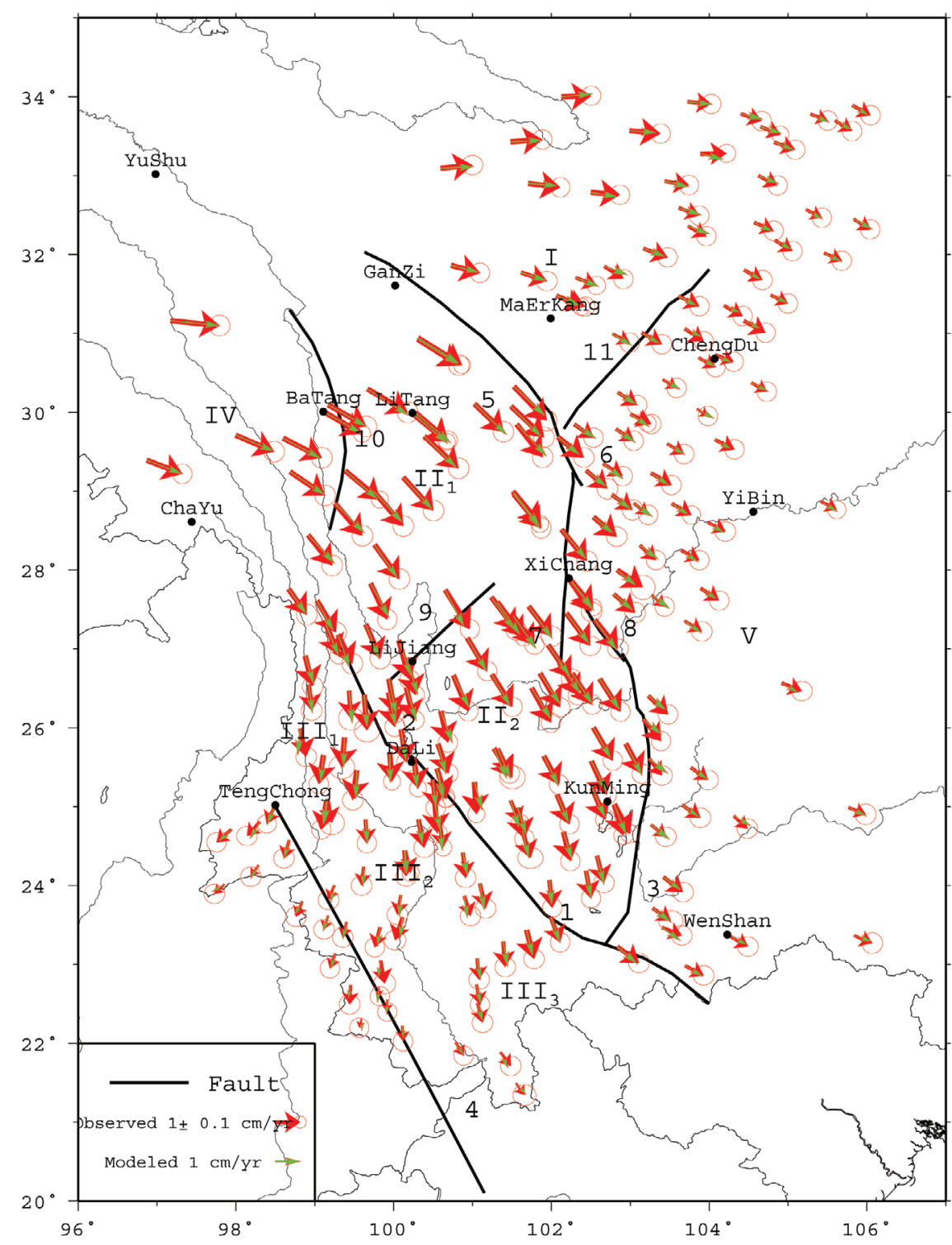

Fig. 2. The fitted velocity field from the heterogeneous strain rate model based on TLS (TLS-HSM). The red arrow denotes the observed velocity, and the green denotes the fitted velocity. GPS velocity field is with respect to the Eurasian plate. 
in the coefficient matrix. Then we use the LS-HSM result as the initial value for the TLS inversion, getting the $\mathrm{K}$ matrix in Eq. (10). Using 249 high-precision GPS horizontal velocities we estimate the motion parameters and strain rate parameters of 8 blocks (sub-blocks). The estimated block parameters for the Sichuan-Yunnan region in Eq. (3) are shown in Table 1. Furthermore, we can obtain other strain rate parameters, such as the surface dilatation rate, maximum shear strain rate, principal strain rates, principal direction and two shear components. The main strain rate parameters of each block in the block center are documented in Table 2. The fitted velocity field from the TLS-HSM is

Table 1. Parameters results from TLS-HSM.

\begin{tabular}{|c|c|c|c|c|c|c|c|c|c|}
\hline & & I & II1 & II2 & III1 & III2 & III3 & IV & $\mathbf{V}$ \\
\hline$V_{n 0}$ & {$\left[\mathrm{~mm} \mathrm{yr} \mathbf{~ r}^{-1}\right]$} & -2.6593 & -13.7297 & -12.5016 & -10.2096 & -7.7806 & -9.1674 & -7.3073 & -4.7790 \\
\hline $\operatorname{RMSE}\left(V_{n 0}\right)$ & {$\left[\mathrm{mm} \mathrm{yr}^{-1}\right]$} & 0.2881 & 0.4340 & 0.2628 & 0.5014 & 0.5127 & 0.4222 & 0.1854 & 0.2369 \\
\hline$V_{e 0}$ & {$\left[\mathbf{m m ~} \mathbf{y r}^{-1}\right]$} & 9.5471 & 10.9161 & 6.8429 & -1.7587 & -0.9396 & 1.5722 & 15.3250 & 6.9407 \\
\hline $\operatorname{RMSE}\left(V_{e 0}\right)$ & {$\left[\mathrm{mm} \mathrm{yr}^{-1}\right]$} & 0.2887 & 0.4340 & 0.2658 & 0.5259 & 0.5375 & 0.4475 & 0.1854 & 0.2414 \\
\hline$A_{0}$ & {$\left[10^{-9} \mathrm{yr}^{-1}\right]$} & 12.3170 & 10.4373 & -7.4603 & -16.8587 & -12.9678 & -22.0477 & 19.3975 & 2.2737 \\
\hline $\operatorname{RMSE}\left(A_{0}\right)$ & {$\left[10^{-9} \mathrm{yr}^{-1}\right]$} & 1.7229 & 1.4334 & 1.3045 & 4.9447 & 5.5385 & 3.7379 & 2.1732 & 0.6240 \\
\hline$A_{1}$ & {$\left[10^{-12} \mathrm{yr}^{-1}\right]$} & 23.6764 & 49.0685 & 42.4182 & 87.7447 & 74.6054 & -7.4869 & 140.6100 & 10.2531 \\
\hline $\operatorname{RMSE}\left(A_{1}\right)$ & {$\left[10^{-12} \mathrm{yr}^{-1}\right]$} & 36.8845 & 25.9072 & 19.4526 & 95.4814 & 111.7810 & 107.3641 & 44.0134 & 4.1968 \\
\hline$A_{2}$ & {$\left[10^{-12} \mathrm{yr}^{-1}\right]$} & -53.7026 & -9.5973 & 60.6157 & -72.9402 & -56.4388 & -236.2001 & 101.0575 & -18.2954 \\
\hline $\operatorname{RMSE}\left(A_{2}\right)$ & {$\left[10^{-12} \mathrm{yr}^{-1}\right]$} & 15.8905 & 35.9058 & 20.8835 & 110.5316 & 115.6791 & 172.7239 & 54.1947 & 5.6288 \\
\hline $\boldsymbol{B}_{0}$ & {$\left[10^{-9} \mathrm{yr}^{-1}\right]$} & -14.6508 & -7.9849 & 14.2379 & 23.6844 & 6.5336 & 10.5121 & -2.3725 & -7.6154 \\
\hline $\operatorname{RMSE}\left(B_{0}\right)$ & {$\left[10^{-9} \mathrm{yr}^{-1}\right]$} & 1.4220 & 2.9064 & 1.9070 & 7.7054 & 11.8601 & 10.2972 & 2.7633 & 2.0956 \\
\hline$B_{1}$ & {$\left[10^{-12} \mathrm{yr}^{-1}\right]$} & -11.1367 & -110.4908 & -53.5904 & -163.1560 & 17.8591 & 136.5703 & -142.5893 & 1.4167 \\
\hline $\operatorname{RMSE}\left(B_{1}\right)$ & {$\left[10^{-12} \mathrm{yr}^{-1}\right]$} & 16.0457 & 35.7086 & 20.9273 & 114.7676 & 118.4826 & 182.1613 & 54.1947 & 5.7076 \\
\hline$B_{2}$ & {$\left[10^{-12} \mathrm{yr}^{-1}\right]$} & -15.0689 & 92.3267 & -186.2118 & -42.6167 & -335.5172 & 190.7201 & -5.3714 & 82.0502 \\
\hline RMSE $\left(B_{2}\right)$ & {$\left[10^{-12} \mathrm{yr}^{-1}\right]$} & 20.2418 & 91.8005 & 44.5561 & 239.4381 & 354.2778 & 679.2408 & 70.7952 & 32.4555 \\
\hline$C_{0}$ & {$\left[10^{-9} \mathrm{yr}^{-1}\right]$} & 1.4093 & 7.7323 & 11.0368 & -10.5226 & -8.2601 & -16.6021 & -1.3498 & 7.3040 \\
\hline $\operatorname{RMSE}\left(C_{0}\right)$ & {$\left[10^{-9} \mathrm{yr}^{-1}\right]$} & 1.1150 & 1.6228 & 1.1487 & 4.5209 & 6.3582 & 5.2818 & 1.7565 & 1.0688 \\
\hline$C_{1}$ & {$\left[10^{-12} \mathbf{y r}^{-1}\right]$} & -43.3163 & -13.3153 & -1.5791 & 114.0194 & 70.5040 & -41.4453 & -38.9620 & -8.2853 \\
\hline RMSE $\left(C_{1}\right)$ & {$\left[10^{-12} \mathrm{yr}^{-1}\right]$} & 20.0922 & 22.1140 & 14.3291 & 74.3603 & 82.1385 & 103.3423 & 34.9397 & 3.5407 \\
\hline$C_{2}$ & {$\left[10^{-12} \mathrm{yr}^{-1}\right]$} & -4.1320 & 16.0502 & -48.0840 & 60.3314 & 26.1298 & 661.1534 & -173.5562 & -63.5714 \\
\hline RMSE $\left(C_{2}\right)$ & {$\left[10^{-12} \mathrm{yr}^{-1}\right]$} & 12.7942 & 49.4127 & 24.3322 & 130.4197 & 180.1204 & 334.8042 & 44.5434 & 16.2006 \\
\hline$D_{0}$ & {$\left[10^{-9} \mathrm{yr}^{-1}\right]$} & -8.5986 & -20.5110 & -11.1536 & -17.2035 & -14.0877 & -13.3827 & -26.4315 & 7.2359 \\
\hline $\operatorname{RMSE}\left(D_{0}\right)$ & {$\left[10^{-9} \mathrm{yr}^{-1}\right]$} & 1.1159 & 1.6241 & 1.1488 & 4.5285 & 6.3639 & 5.2768 & 1.7590 & 1.0686 \\
\hline
\end{tabular}


$\mathrm{Xu}$ et al.

Table 2. Strain rate parameters in the center of blocks from TLS-HSM.

\begin{tabular}{|c|c|c|c|c|c|c|c|c|c|}
\hline & & $\mathbf{I}$ & II1 & II 2 & III1 & III2 & III3 & IV & $\mathbf{V}$ \\
\hline$\omega$ & {$\left[{ }^{0} \mathbf{M y r}^{-1}\right]$} & -0.4927 & -1.1752 & -0.6391 & -0.9857 & -0.8072 & -0.7668 & -1.5144 & 0.4146 \\
\hline RMSE $(\omega)$ & {$\left[{ }^{0} \mathrm{Myr}^{-1}\right]$} & 0.0639 & 0.0931 & 0.0659 & 0.2595 & 0.3646 & 0.3023 & 0.1008 & 0.0612 \\
\hline$\dot{\Delta}$ & {$\left[10^{-9} \mathrm{yr}^{-1}\right]$} & -2.3338 & 2.4525 & 6.7776 & 6.8257 & -6.4342 & -11.5356 & 17.0250 & -5.3417 \\
\hline $\operatorname{RMSE}(\dot{\Delta})$ & {$\left[10^{-9} \mathrm{yr}^{-1}\right]$} & 2.2330 & 3.2393 & 2.3105 & 9.1480 & 13.0839 & 10.9594 & 3.5130 & 2.1867 \\
\hline$\dot{\gamma}_{\text {max }}$ & {$\left[10^{-9} \mathrm{yr}^{-1}\right]$} & 27.1148 & 24.0527 & 30.9525 & 45.6798 & 25.5582 & 46.5045 & 21.9367 & 17.6405 \\
\hline $\operatorname{RMSE}\left(\dot{\gamma}_{\max }\right)$ & {$\left[10^{-9} \mathrm{yr}^{-1}\right]$} & 2.2348 & 3.2381 & 2.2971 & 9.1922 & 13.1056 & 10.6577 & 3.5182 & 2.1496 \\
\hline$\dot{\varepsilon}_{1}$ & {$\left[10^{-9} \mathrm{yr}^{-1}\right]$} & 12.3905 & 13.2526 & 18.8651 & 26.2527 & 9.5620 & 17.4844 & 19.4809 & 6.1494 \\
\hline RMSE $\left(\dot{\varepsilon}_{1}\right)$ & {$\left[10^{-9} \mathrm{yr}^{-1}\right]$} & 1.6877 & 1.2624 & 1.8353 & 8.2260 & 12.3235 & 8.7965 & 2.2977 & 1.1177 \\
\hline$\dot{\boldsymbol{\varepsilon}}_{2}$ & {$\left[10^{-9} \mathrm{yr}^{-1}\right]$} & -14.7243 & -10.8001 & -12.0875 & -19.4270 & -15.9962 & -29.0201 & -2.4558 & -11.4911 \\
\hline $\operatorname{RMSE}\left(\dot{\varepsilon}_{2}\right)$ & {$\left[10^{-9} \mathrm{yr}^{-1}\right]$} & 1.4635 & 2.9826 & 1.3926 & 4.0527 & 4.4276 & 6.2825 & 2.6609 & 1.8579 \\
\hline$\theta$ & {$\left[{ }^{\circ}\right]$} & -2.9833 & -20.0061 & 22.7456 & -13.7165 & -20.1343 & -22.7807 & 3.5345 & -27.9516 \\
\hline RMSE $(\theta)$ & {$\left[{ }^{\circ}\right]$} & 2.3562 & 3.8702 & 2.1389 & 5.6520 & 14.2417 & 6.6890 & 4.5876 & 3.5313 \\
\hline$\dot{\gamma}_{1}$ & {$\left[10^{-9} \mathrm{yr}^{-1}\right]$} & 26.9679 & 18.4222 & -21.6983 & -40.5431 & -19.5015 & -32.5599 & 21.7700 & 9.8891 \\
\hline $\operatorname{RMSE}\left(\dot{\gamma}_{1}\right)$ & {$\left[10^{-9} \mathrm{yr}^{-1}\right]$} & 2.2349 & 3.2420 & 2.3106 & 9.1631 & 13.0952 & 10.9499 & 3.5181 & 2.1864 \\
\hline$\dot{\gamma}_{2}$ & {$\left[10^{-9} \mathrm{yr}^{-1}\right]$} & 2.8186 & 15.4647 & 22.0736 & -21.0452 & -16.5201 & -33.2043 & -2.6996 & 14.6080 \\
\hline $\operatorname{RMSE}\left(\dot{\gamma}_{2}\right)$ & {$\left[10^{-9} \mathrm{yr}^{-1}\right]$} & 2.2299 & 3.2455 & 2.2975 & 9.0418 & 12.7164 & 10.5636 & 3.5130 & 2.1375 \\
\hline
\end{tabular}

Note: I: the Maerkang block; III: the north-western Sichuan sub-block; II2: the central Yunnan sub-block; III1: the Baoshan sub-block; III2: the Jinggu subblock; III3: the Mengla sub-block; IV: the Qiangtang block; V: the southern China block. $\omega$ is the rotation rate about the local vertical (negative direction is clockwise); $\dot{\Delta}$ is the surface dilatation rate; $\dot{\gamma}_{\max }$ is the maximum shear strain rate; $\dot{\varepsilon}_{1}$ and $\dot{\varepsilon}_{2}$ are the principal strain rates; $\theta$ is the principal direction; $\dot{\gamma}_{1}$ is one shear component; $\dot{\gamma}_{2}$ is another shear component.

shown in the Fig. 2. The strain rates inferred from the TLSHSM for a discrete $0.5^{\circ} \times 0.5^{\circ}$ grid are shown in Fig. 3 . Diagrams of the discrete grid point maximum shear strain rate and areal strain rate (dilatation) from the TLS-HSM are shown in Figs. 4 and 5, respectively. The strain rates and rotation inferred from the TLS-HSM for the block center are shown in Figs. 6 and 7, respectively.

The differences of block motion and strain parameters between the least squares uniform strain (rate) model (LS-USM) (Xu and Wen 2007) and TLS-HSM are shown in Fig. 8. If the difference ('+') is positive, it denotes that the component of velocity and the strain rate parameters in the LS-USM is larger than that in the TLS-HSM. If the difference ('o') is positive, it denotes that the component of the mean square velocity and strain rate parameter error in the LS-USM is larger than that in the TLS-HSM. As shown in Fig. 8, except for the velocity of the Jinggu sub-block, all the velocities and the strain rate parameters of the blocks obtained from TLS-HSM have a higher accuracy than that from the LS-USM. We can see that TLS-HSM is more suitable for the crustal motion and deformation in the region than LS-USM.

\section{DISCUSSION}

\subsection{Strain Rates from TLS-HSM}

The strain rates of the 8 blocks (sub-blocks) are documented in Table 2 and Figs. 3 through 9. The TLS-HSM is a heterogeneous strain model, which can describe strain features in different parts of a block.

In the Maerkang block, the surface dilatation rate in the block center from TLS-HSM is smaller than that from LSUSM. Near the northern segment of the Xianshuihe Fault, the principal strain rate is larger than the other part (Fig. 3). The maximum shear strain rate is small (Fig. 4). The rotation strain rate is clockwise (Fig. 7). The block extends in 
a W-E direction with compression in a N-S direction, and extends in a NE-SW direction with compression in a NWSE direction.

The principal dilatation strain rate, principal contraction strain rate, maximum shearing strain rate and surface dilation rate in the north-western Sichuan subblock center are $13.2526 \pm 1.2624,-10.8001 \pm 2.9826,24.0527 \pm 3.2381$, $2.4525 \pm 3.2393 \times 10^{-9} \mathrm{yr}^{-1}$ (with a $95 \%$ confidence probability), respectively. The block extends in a W-E direction with contraction in a N-S direction, and extends in a NE-SW direction with contraction in a NW-SE direction. The surface dilatation rate is a small positive value (Fig. 5), so the block deforms without changing the area very much. The rotation of the block is clockwise (Fig. 7).

The principal dilatation strain rate and the principal contraction strain rate of the central Yunnan sub-block in the west are larger than in the eastern part (Fig. 3). The maximum shear strain rate of the eastern part is larger than the western part (Fig. 4). The surface dilatation rate is a small positive value, but there is an unusually large positive value in the part where the Anninghe Fault and the Zemuhe Fault converge (Fig. 5). The rotation of the block is clockwise (Fig. 7). The block extends in the N-S direction with contraction in a W-E direction, and extends in a NE-SW direction with contraction in a NW-SE direction.

The Baoshan sub-block, the Jinggu sub-block and the Mengla sub-block all extend in a N-S direction with contraction in a W-E direction, and extend in a NW-SE direction with contraction in a NE-SW direction (Figs. 4 and 6). The detailed spatial patterns of principal strain of these three sub-blocks are: (1) the Baoshan sub-block is mainly S-N contraction; (2) the Jinggu sub-block is mainly an E-W extensional; (3) the S-N contractional principal strain and E-W extensional principal strain in the Mengla sub-block are generally equal with each other in LS-USM. There is a small abnormal part with smaller maximum shear strain rate in the Jinggu sub-block, while, a small abnormal part with larger maximum shear strain rate in the southeastern part of Mengla sub-block (Fig. 4). The surface dilatation rate gradually becomes smaller from the Baoshan sub-block to the Mengla sub-block (Fig. 5). The principal dilatation strain rate, the principal contraction strain rate, the maximum shearing strain rate and the area strain rate of these blocks are listed in Table 2 .

As shown in Fig. 4, the shear strain rate in the vicinity of the Red River fault can be very high. The high shearing strain rate and area dilatation strain rate in the SichuanYunnan rhombic block and the central Yunnan sub-block indicate that the two blocks play a key role in the research of continental tectonic deformation (Figs. 4 and 5). The northwestern Sichuan sub-block, central Yunnan sub-block, Baoshan sub-block and Qiangtang blocks are in zones with an area dilatation strain rate. The Maerkang sub-block, Jinggu sub-block, Mengla sub-block and south China block are in zones with an area contraction strain rate (Fig. 5). In LS-USM, the Jinggu sub-block and Mengla sub-block are in zones with an area dilatation strain rate and the Baoshan sub-block is in a zone with an area contraction strain rate.

Because of the heterogeneous distribution of GPS velocity data, the results around the edge of studied area are less certain. From the maximum shear strain rate (Fig. 4), the southern segment of the Longmenshan Fault is in a higher maximum shear strain rate area compared with the surrounding area. From the area strain rate (Fig. 5), the southern segment of the Longmenshan Fault is in a negative area strain rate area (areal contraction), while the domain around the Xianshuihe fault is in a positive area strain rate area (areal dilatation). Therefore, the gradient of an area strain rate is very large, and may be related to thrust earthquakes. The 12 May 2008 Wenchuan $M_{w} 7.9$ earthquake occurred along the Longmen Shan fault zone, so there may be some relationship for the earthquake with area strain rate and maximum shear strain rate, Meng et al. (2008) also obtained the same results. Effective strain rates reach $30-34 \times 10^{-8} \mathrm{yr}^{-1}$ near the southeastern section of the Xianshuihe fault and close to the southern end of the Anninghe fault. Along most of the Longmenshan fault, effective strain rates are less than

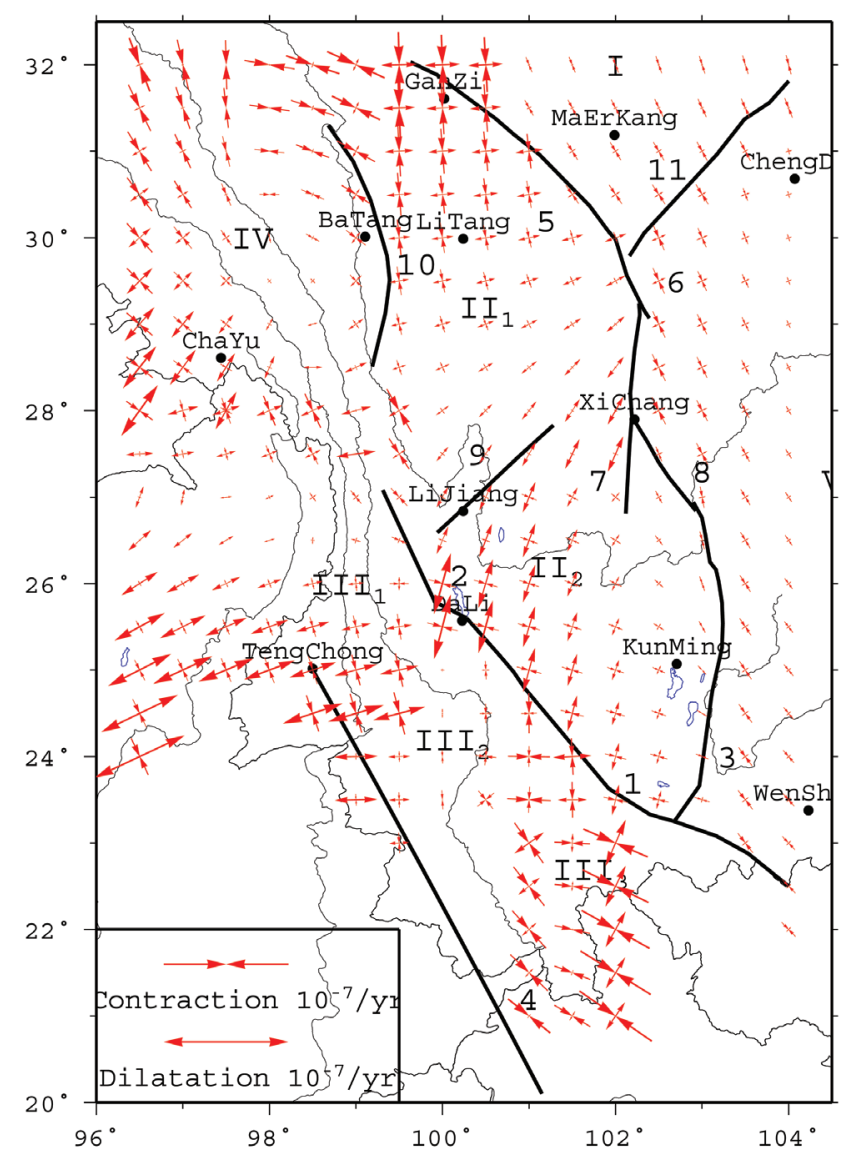

Fig. 3. The strain rates inferred from the TLS-HSM for a discrete $0.5^{\circ}$ $\times 0.5^{\circ}$ grid. 


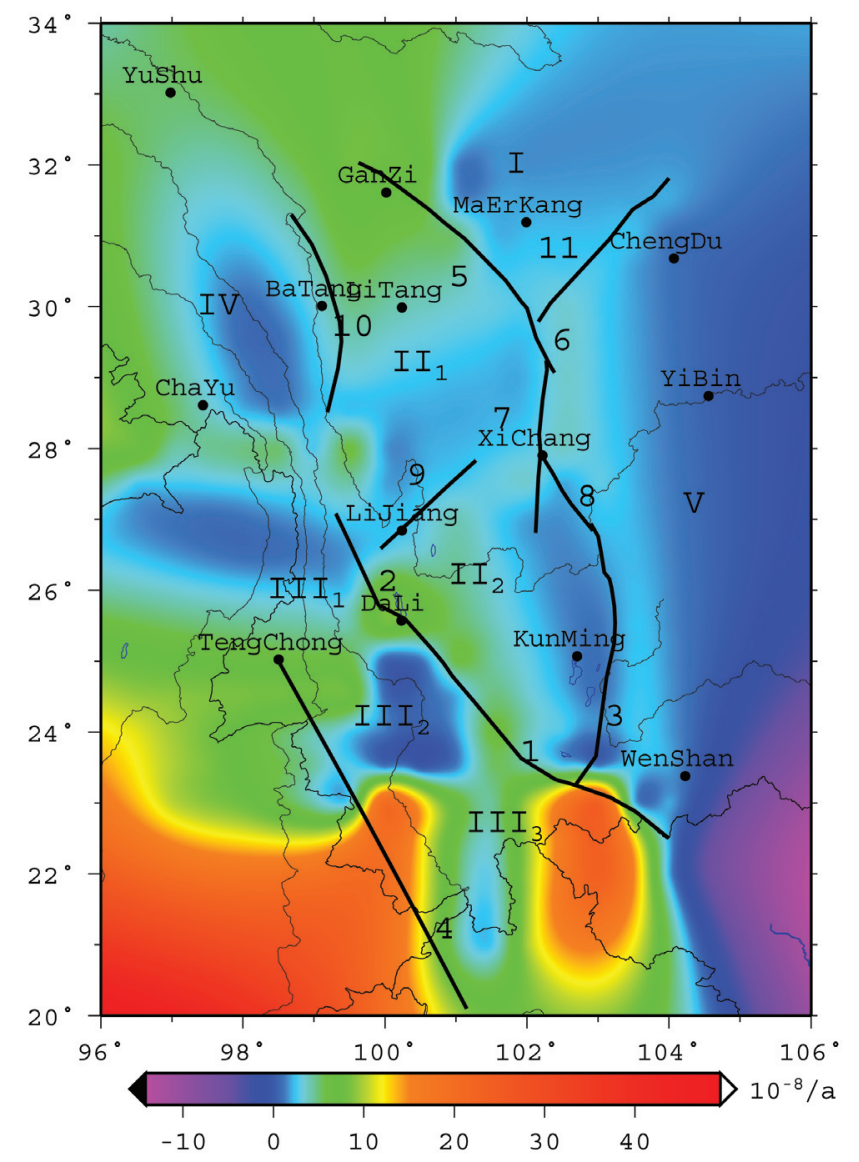

Fig. 4. Diagram of the discrete grid point maximum shear strain rate from the heterogeneous strain rate model based on TLS (TLS-HSM).

$10^{-7} \mathrm{yr}^{-1}$. The epicenter of the recent $\mathrm{M}_{\mathrm{s}} 8.0$ earthquake appears at a domain of effective strain rates between $10^{-8}$ and $10^{-7} \mathrm{yr}^{-1}$, with relatively higher strain rate gradients (Meng et al. 2008). We also find in our results, like Vergnolle et al. (2007) and Meng et al. (2008), that there is also a domain of localized strain rates around the Xianshuihe fault (Figs. 4 and 5), but our domain is not very clear.

By comparing Fig. 4 with the earthquake focal mechanisms from the Harvard CMT catalog, 1976 - 2003 (Shen et al. 2005; Fig. 2), we can see that in the Red River Fault area the maximum shear strain rate from the TLS-HSM is higher and has experienced many earthquakes here. While, the deformation along the southern segment of the Red River fault does not appear to be significant at present (Shen et al. 2005), in the Red River Fault area, the strain rates inferred from the TLS-HSM are also very high.

Due to the India-Eurasian collision along a N-NE trend and eastward reduction of geopoential energy in the Tibetan Plateau as well as obstructing of the rigid southern China block to its east, the mass in this south-eastern borderland flows in a clockwise manner around the so-called Eastern Himalayan Syntaxis (EHS) (Wang et al. 2008). In these blocks (or sub-blocks) such as Qiangtang block, Maerkang

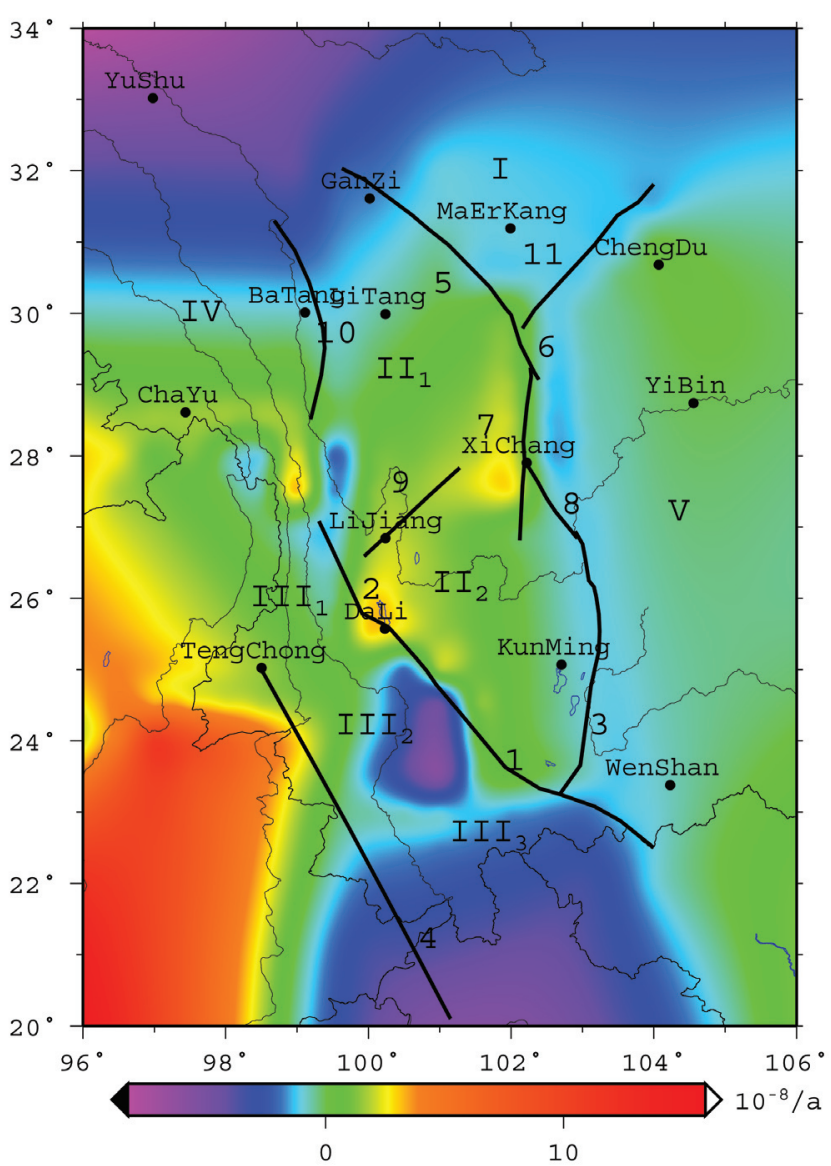

Fig. 5. Diagram of the discrete grid point area strain rate from the heterogeneous strain rate model based on TLS (TLS-HSM).

block, north-western Sichuan sub-block, central Yunnan sub-block and Baoshan sub-block, the dilation strain rate is predominated, which is consistent with the point that mass flow in this region extensionally spreads (e.g., Gan et al. 2007).

\subsection{Block Motion from TLS-HSM}

Using the inversion results from TLS-HSM, we modeled the GPS velocity field (Fig. 2). The modeled velocity data is consistent with the observed velocity data. With respect to the Eurasia plate, Qiangtang block, north-western Sichuan sub-block, central Yunnan sub-block, Baoshan sub-block, Jinggu sub-block and Mengla show clockwise sub-block rotations. Figure 9 shows the model velocity field at the centroid of each block (or sub-block) from the TLS-HSM estimator. With respect to the Eurasian plate, the Maerkang block moves in the direction of $\mathrm{N} 105.6 \pm 1.7^{\circ} \mathrm{E}$ at a rate of $9.9 \pm 0.4 \mathrm{~mm} \mathrm{yr}^{-1}$ (with a $95 \%$ confidence probability); the north-western Sichuan sub-block moves in the direction of $\mathrm{N} 141.5 \pm 1.4^{\circ} \mathrm{E}$ at a rate of $17.5 \pm 0.6 \mathrm{~mm}$ $\mathrm{yr}^{-1}$ (with a $95 \%$ confidence probability); the central Yunnan sub-block moves in the direction of $\mathrm{N} 151.3 \pm 1.1^{\circ} \mathrm{E}$ at 


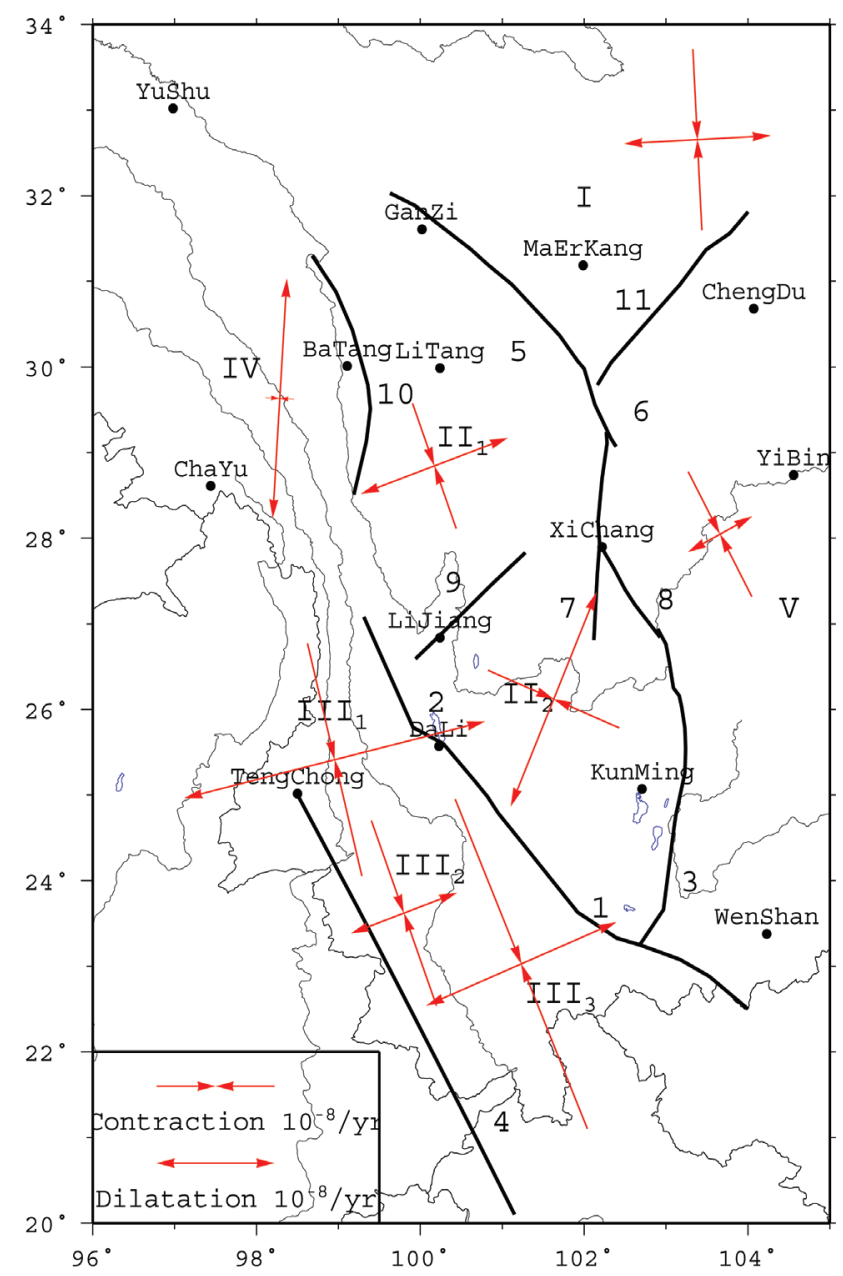

Fig. 6. The strain rates inferred from the heterogeneous strain rate model based on TLS (TLS-HSM) for the block center.

a rate of $14.3 \pm 0.4 \mathrm{~mm} \mathrm{yr}^{-1}$ (with a $95 \%$ confidence probability); the Baoshan sub-block moves in the direction of $\mathrm{N} 189.8 \pm 2.8^{\circ} \mathrm{E}$ at a rate of $10.4 \pm 0.7 \mathrm{~mm} \mathrm{yr}^{-1}$ (with a 95\% confidence probability); the Jinggu sub-block moves in the direction of $\mathrm{N} 186.9 \pm 3.7^{\circ} \mathrm{E}$ at a rate of $7.8 \pm 0.7 \mathrm{~mm}$ $\mathrm{yr}^{-1}$ with a $95 \%$ confidence probability); the Mengla subblock moves in the direction of $\mathrm{N} 170.3 \pm 2.6^{\circ} \mathrm{E}$ at a rate of $9.3 \pm 0.6 \mathrm{~mm} \mathrm{yr}^{-1}$ (with a $95 \%$ confidence probability); the Qiangtang block moves in the direction of $\mathrm{N} 115.5 \pm 0.6^{\circ} \mathrm{E}$ at a rate of $17.0 \pm 0.3 \mathrm{~mm} \mathrm{yr}^{-1}$ (with $95 \%$ confidence probability). The southern China block moves in the direction of $\mathrm{N} 124.5 \pm 1.6^{\circ} \mathrm{E}$ at a rate of $8.4 \pm 0.3 \mathrm{~mm} \mathrm{yr}^{-1}$ (with a $95 \%$ confidence probability) with respect to the Eurasia plate, which is also consistent with less than $10 \mathrm{~mm} \mathrm{yr}^{-1}$ derived from 'the continuous deformation' hypothesis (England and Houseman 1986; Houseman and England 1993; Molnar et al. 1993; England and Molnar 1997). Movement of each of the blocks (or sub-blocks) calculated by TLS-HSM are different from those estimated by LS-USM. For example, the Mengla sub-block rotates clockwise with estimator based

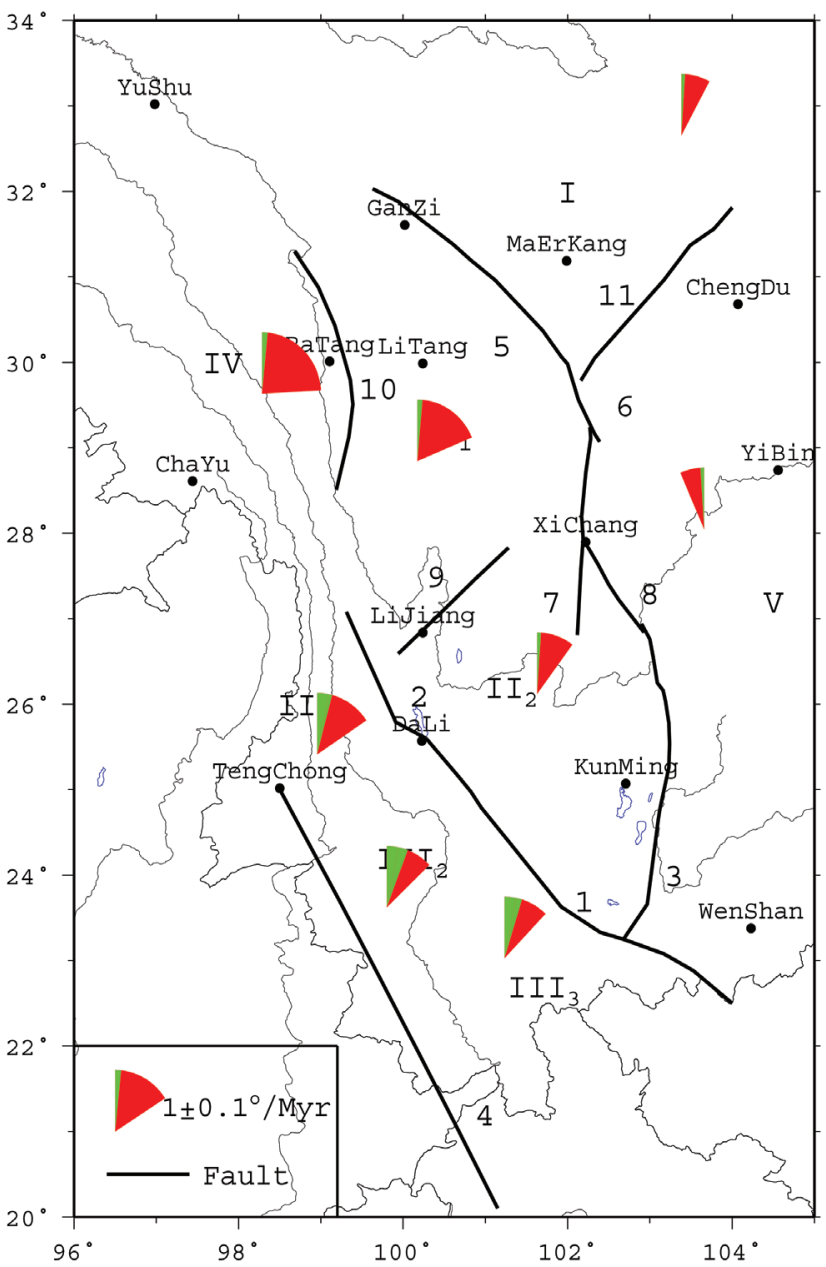

Fig. 7. The rotation inverted from the heterogeneous strain rate model based on TLS (TLS-HSM) for the block center.

upon the TLS-HSM estimator and rotates counterclockwise based upon the LS-USM estimator. Each of the rotations of the other blocks (or sub-blocks) behaves in the same manner as that of the Mengla sub-block.

\subsection{Comparisons between TLS-HSM and LS-HSM}

Before the inversion of the Sichuan-Yunnan region, we conducted synthetic examples to test the importance of the TLS estimator. The results from TLS considering the error of coefficient matrix are more near to the true value than LS. The difference between LS results and the true value are larger. The relative error for the LS estimation is between $0.55 \%$ and $10.21 \%$, while the relative error for the TLS estimation is between $0.52 \%$ and $9.61 \%$. The difference of precision between LS and TLS is not very large. We can see that the errors in coordinates occur at the millimeter level (e.g., $3.16 \mathrm{~mm}$ ) and can cause some changes in the strain rates.

Generally, strain rate parameters estimated by TLSHSM are more precise than those estimated by LS-HSM 

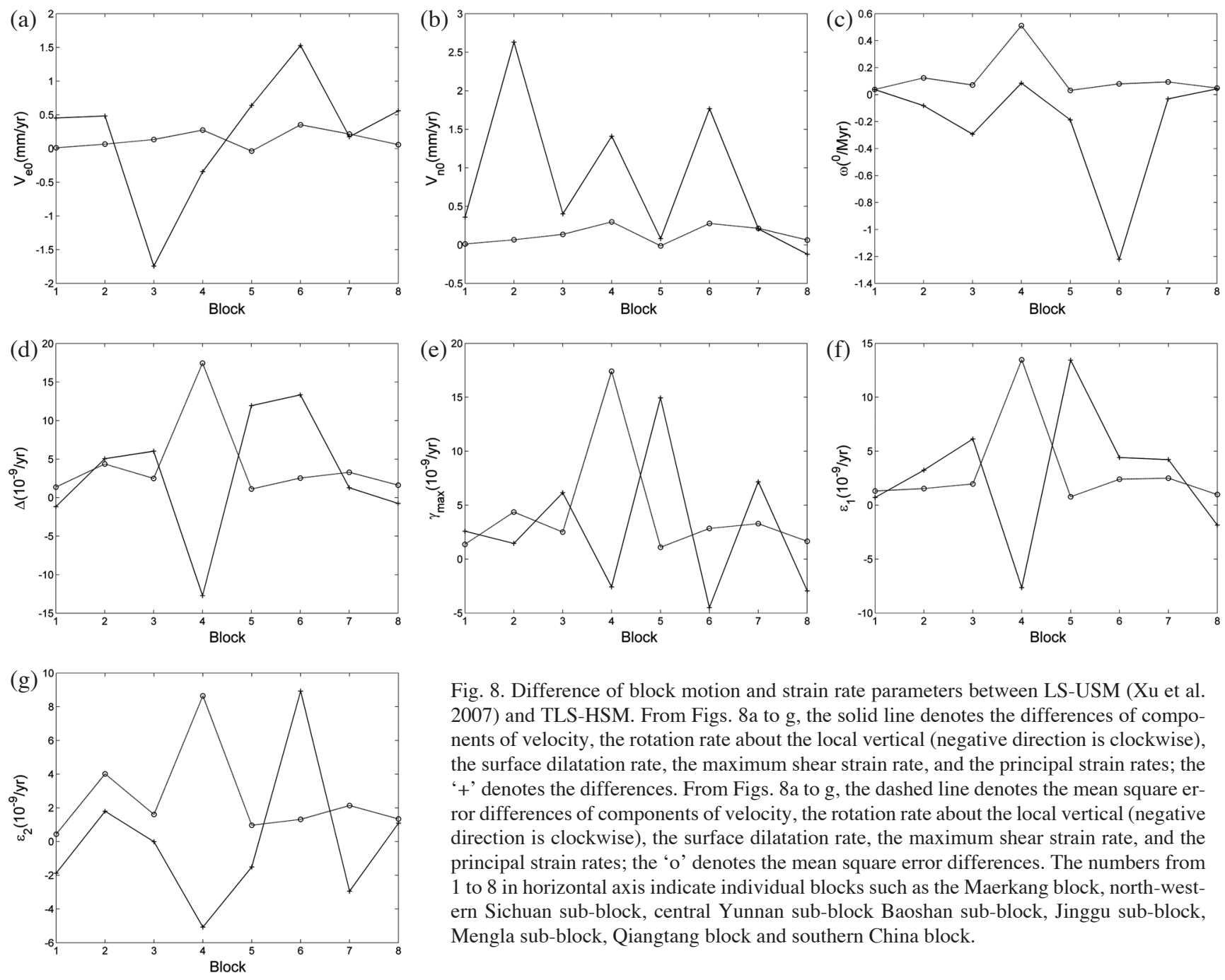

Fig. 8. Difference of block motion and strain rate parameters between LS-USM (Xu et al. 2007) and TLS-HSM. From Figs. 8a to g, the solid line denotes the differences of components of velocity, the rotation rate about the local vertical (negative direction is clockwise), the surface dilatation rate, the maximum shear strain rate, and the principal strain rates; the ' + ' denotes the differences. From Figs. 8a to g, the dashed line denotes the mean square error differences of components of velocity, the rotation rate about the local vertical (negative direction is clockwise), the surface dilatation rate, the maximum shear strain rate, and the principal strain rates; the 'o' denotes the mean square error differences. The numbers from 1 to 8 in horizontal axis indicate individual blocks such as the Maerkang block, north-western Sichuan sub-block, central Yunnan sub-block Baoshan sub-block, Jinggu sub-block, Mengla sub-block, Qiangtang block and southern China block.

for most blocks (or sub-blocks) (Table 3). For the Maerkang block, north-western Sichuan sub-block, Baoshan subblock, Jinggu sub-block, Qiangtang block and southern China block, their posterior mean square errors of unit weight estimated by LS-HSM are smaller than those estimated by TLS-HSM. However, there are two sub-blocks, the central Yunnan sub-block and the Mengla sub-block for which parameters estimated by TLS are less precise than those estimated by LS. The key point of using TLS method is the cofactor matrix in Eq. (14). It is the difference between LS method and TLS method. So the distribution and number of GPS stations are very important to the determination of crustal strain parameters. When the coefficient matrix has full rank and all rows of the augmented matrix made of observations and coefficient matrix are independently and identically distributed with zero mean and identity matrix of covariance: for TLS, the sum of squared perpendicular distances from each column of the augmented matrix to its estimated space is minimized, and each column of the augmented matrix is approximated by its orthogonal projection onto that subspace; while for LS, the solution is obtained by projecting observation vector orthogonally onto the space of the coefficient matrix. That is the reason why TLS gives a better fit. It is easily showed that the TLS correction is always smaller in the norm than the LS correction (van Huffel and Vandewalle 1991). When the TLS is weighted, the fit may be affected by the weight. The difference between posterior estimates mean square error of unit weight obtained from LS-HSM and TLS-HSM for the blocks are shown in Table 3 and Fig. 10.

\section{CONCLUSIONS}

In this paper, the elastic block motion is regarded as a block motion with linear strain and rotation rather than the pure rigid motion. Unlike LS-USM (Savage et al. 2001, 2004; Gan et al. 2007; Xu and Wen 2007), which can only describe the strain feature in the whole block without being able to explore the details of the block, the spatial pattern of strain field is considered to be linearly coordinate-dependent 


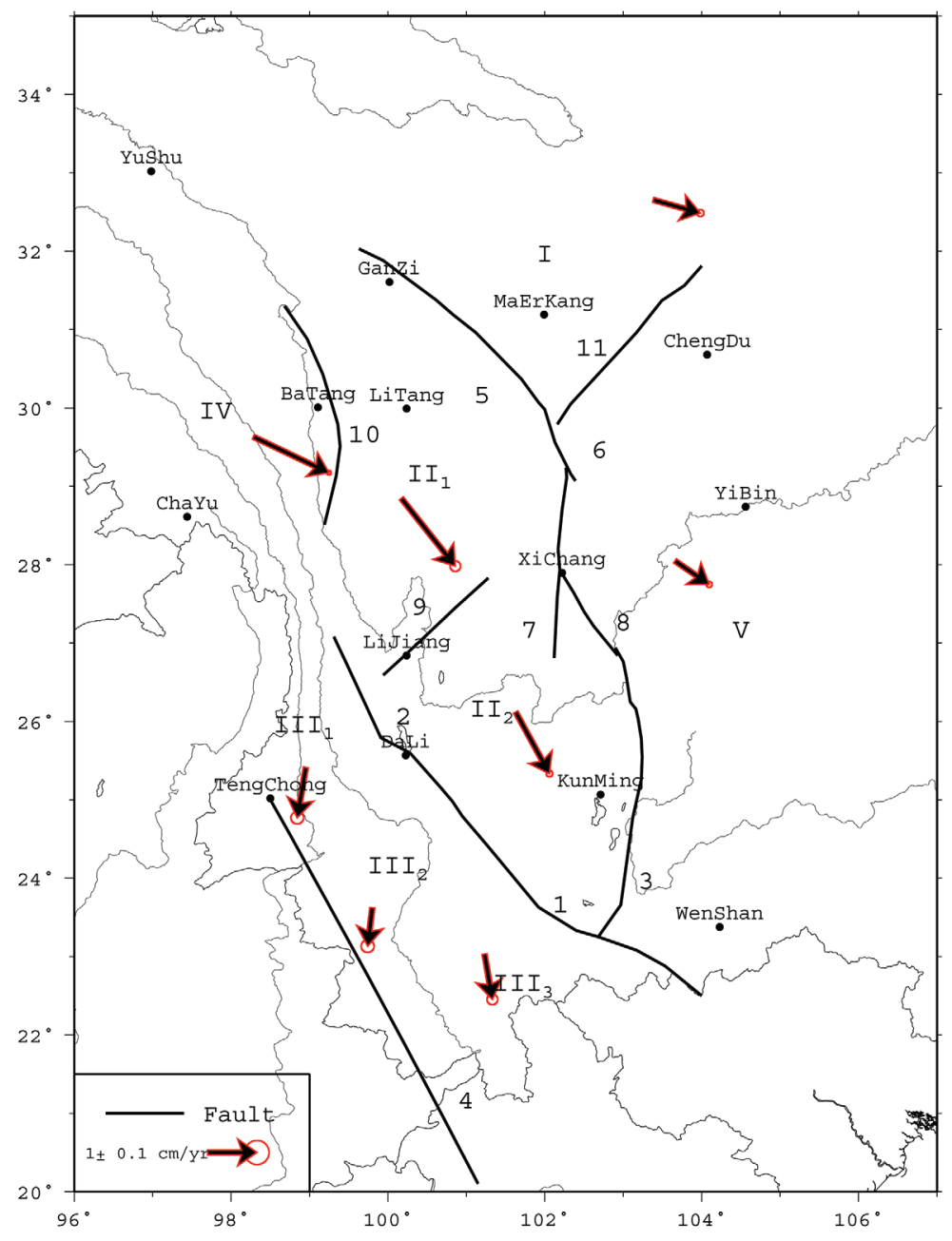

Fig. 9. The motion of blocks inferred from the heterogeneous strain rate model based on TLS (TLS-HSM). The motion is with respect to the Eurasian plate. The error ellipses represent a 95\% confidence ellipse.

Table 3. Posterior root mean square error of unit weight obtained from LS-HSM and TLS-HSM.

\begin{tabular}{cc|cccccccc}
\hline & & I & II1 & II2 & III1 & III2 & III3 & IV & V \\
\hline LS-HSM & {$\left[\mathbf{1 0}^{-7} \mathbf{y r}^{-1}\right]$} & 8.5411 & 9.4582 & 9.0339 & 11.7156 & 13.0434 & 8.2971 & 2.3042 & 9.9785 \\
TLS-HSM & {$\left[\mathbf{1 0}^{-7} \mathbf{y r}^{-1}\right]$} & 8.5386 & 9.4382 & 9.0452 & 11.7102 & 13.0374 & 8.3181 & 2.3034 & 9.9361 \\
LS-TLS & {$\left[\mathbf{1 0}^{-9} \mathbf{y r}^{-1}\right]$} & 0.2500 & 2.0000 & -1.1300 & 0.5400 & 0.6000 & -2.1000 & 0.0800 & 4.2400 \\
\hline
\end{tabular}

Note: I: the Maerkang block; III: the north-western Sichuan sub-block; II2: the central Yunnan sub-block; III1: the Baoshan sub-block; III2: the Jinggu sub-block; III3: the Mengla sub-block; IV: the Qiangtang block; V: the southern China block.

and thus it is heterogeneous in our TLS-HSM which can describe a strain feature in a different part of the block. Using 249 high-precision GPS horizontal velocities and the TLSHSM, we obtain the strain features of the Sichuan-Yunnan region. The results in this paper have higher accuracy than that in LS-USM, although the same data are used. Therefore, TLS-HSM is preferred to delineate crustal motion and deformation in this region compared with LS-USM. Small errors in site coordinates can have a significant impact on strain estimates, especially where sites are located close together.

The principal dilation strain rate, principal contraction strain rate, maximum shearing strain rate and surface dilation rate estimated by TLS-HSM in northwestern Sichuan- 


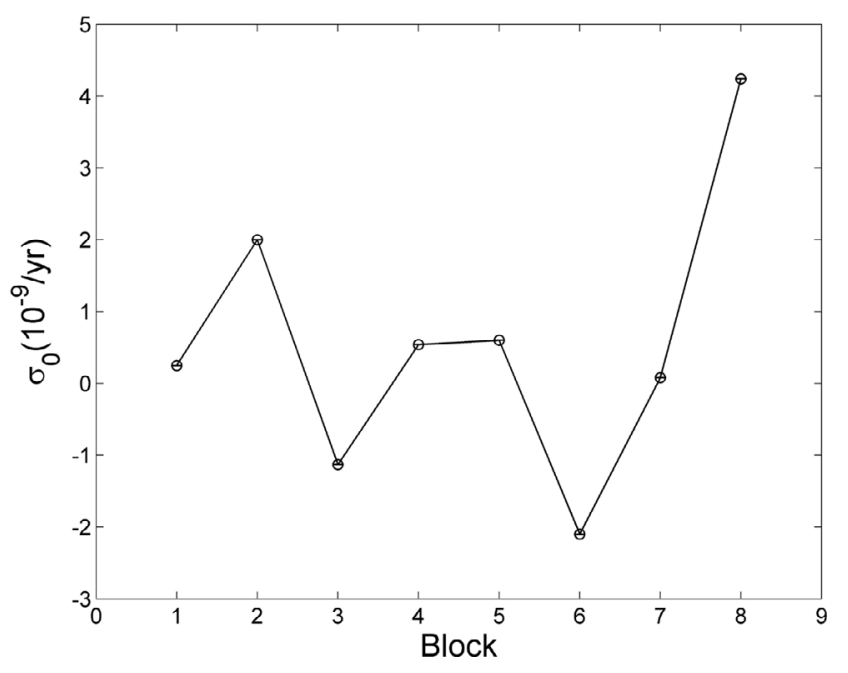

Fig. 10. Difference between posterior estimates mean square error of unit weight obtained from LS-HSM and TLS-HSM. The numbers from 1 to 8 on the horizontal axis indicate individual blocks such as the Maerkang block, north-western Sichuan sub-block, central Yunnan sub-block, Baoshan sub-block, Jinggu sub-block, Mengla sub-block, Qiangtang block and southern China block.

Yunnan sub-block are 13.2526 $\pm 1.2624,-10.8001 \pm 2.9826$, $24.0527 \pm 3.2381,2.4525 \pm 3.2393 \times 10^{-9} \mathrm{yr}^{-1}$ (with a $95 \%$ confidence probability), respectively. Those in the southeastern Sichuan-Yunnan sub-block are 18.8651 \pm 1.8353 , $-12.0875 \pm 1.3926,30.9525 \pm 2.2971$ and $6.7776 \pm 2.3105$ $\times 10^{-9} \mathrm{yr}^{-1}$ (with a $95 \%$ confidence probability), respectively. The motion velocity of the north-western Sichuan sub-block is $17.5 \pm 0.6 \mathrm{~mm} \mathrm{yr}^{-1}$ (with a $95 \%$ confidence probability) and is the highest in the Sichuan-Yunnan region. The motion velocity of the central Yunnan sub-block also demonstrates a very high rate of $14.3 \pm 0.4 \mathrm{~mm} \mathrm{yr}^{-1}$ (with a $95 \%$ confidence probability), thus, the two sub-blocks play a key role in the research of continental tectonic deformation. Based upon strain rates and their direction (Fig. 6), and the clockwise rotation (Fig. 7) of the Maerkang block, the north-western Sichuan sub-block, central Yunnan sub-block, Baoshan subblock, Jinggu sub-block, Mengla sub-block and Qiangtang block, we can see that our results support the hypothesis of an "eastward broaching stress of geopotential energy of the Plateau and the southeastern Plateau is extruded to eastward." The rotation of the southern China block is counterclockwise, so it obstructs eastward mass flow.

Acknowledgements The authors thank Prof. Jeffrey $\mathrm{T}$. Freymueller and one anonymous reviewer for helpful and perceptive comments. The research was supported by the National Natural Science Foundation of China (No. 40874003, 41074007, 41021061, and 40721001), the National Department Public Benefit Research Foundation (Earthquake) (No. 200808080) and the National High Technology Research and Development Program of China (863 Program) (No. 2009AA12Z317) and the Specialized Re- search Fund for the Doctoral Program of Higher Education (No. 20090141110055).

\section{REFERENCES}

Burchfiel, B. C., L. H. Royden, R. D. van der Hilst, B. H. Hager, Z. Chen, R. W. King, C. Li, J. Lü, H. Yao, and E. Kirby, 2008: A geological and geophysical context for the Wenchuan earthquake of 12 May 2008, Sichuan, People's Republic of China. GSA Today, 18, 4-11, doi: 10.1130/GSATG18A.1. [Link]

Dietrich, R., R. Dach, G. Engelhardt, J. Ihde, W. Korth, H.-J. Kutterer, K. Lindner, M. Mayer, F. Menge, H. Miller, C. Müller, W. Niemeier, J. Perlt, M. Pohl, H. Salbach, H.-W. Schenke, T. Schöne, G. Seeber, A. Veit, and C. Völksen, 2001: ITRF coordinates and plate velocities from repeated GPS campaigns in Antarctica - An analysis based on different individual solutions. J. Geodesy, 74, 756-766, doi: 10.1007/s001900000147. [Link]

England, P. and G. Houseman, 1986: Finite Strain Calculations of Continental Deformation 2. Comparison with the India-Asia Collision Zone. J. Geophys. Res., 91, 3664-3676, doi: 10.1029/JB091iB03p03664. [Link]

England, P. and P. Molnar, 1997: The field of crustal velocity in Asia calculated from Quaternary rates of slip on faults. Geophys. J. Int., 130, 551-582, doi: 10. 1111/j.1365-246X.1997.tb01853.x. [Link]

Freymueller, J. T., H. Woodard, S. C. Cohen, R. Cross, J. Elliott, C. Larsen, S. Hreinsdottir, and C. Zweck, 2008: Active deformation processes in Alaska, based on 15 years of GPS measurements. In: Freymueller, J. T., P. J. Haeussler, R. Wesson, and G. Ekstrom, (Eds.), Active Tectonics and Seismic Potential of Alaska, AGU Geophysical Monograph, 179, AGU, Washington DC, $1-42$.

Gan, W., P. Zhang, Z. K. Shen, Z. Niu, M. Wang, Y. Wan, D. Zhou, and J. Cheng, 2007: Present-day crustal motion within the Tibetan Plateau inferred from GPS measurements. J. Geophys. Res., 112, B08416, doi: 10. 1029/2005JB004120. [Link]

Golub, G. H. and C. F. van Loan, 1980: An analysis of the total least squares problem. SIAM J. Numer. Anal., 17, 883- 893, doi: 10.1137/0717073. [Link]

Hao, J. L., W. M. Wang, J. Wang, and Z. X. Yao, 2009: A dislocation model of elastic block for a seismic crustal deformation in Taiwan. Chin. J. Geophys., 52, 12231232.

Houseman, G. and P. England, 1993: Crustal thickening versus lateral expulsion in the India-Asian continental collision. J. Geophys. Res., 98, 12233-12249, doi: 10.1029/93JB00443. [Link]

Larson, K. M., A. Bilich, and P. Axelrad, 2007: Improving the precision of high-rate GPS. J. Geophys. Res., 112, B05422, doi: 10.1029/2006JB004367. [Link] 
Li, Y. X., J. H. Zhang, J. K. He, J. L. Li, Z. Li, L. Q. Guo, Z. F. Zhang, and X. S. Du, 2007: Current-day tectonic motion and intraplate deformation strain field obtained from space geodesy in Pacific Plate. Chin. J. Geophys., 50, 437-447.

Lü, J. N., Z. K. Shen, and M. Wang, 2003: Contemporary crustal deformation and active tectonic block of the Sichuan-Yunnan, China. Seismol. Geol., 25, 543-554.

Markovsky, I. and S. van Huffel, 2007: Overview of total least-squares methods. Signal Process., 87, 2283-2302, doi: 10.1016/j.sigpro.2007.04.004. [Link]

Meng, G., J. Ren, M. Wang, W. Gan, Q. Wang, X. Qiao, and Y. Yang, 2008: Crustal deformation in western Sichuan region and implications for 12 May $2008 M_{s} 8.0$ earthquake. Geochem. Geophys. Geosyst., 9, Q11007, doi: 10.1029/2008GC002144. [Link]

Molnar, P., P. England, and J. Martinod, 1993: Mantle dynamics, uplift of the Tibetan Plateau, and the Indian monsoon. Rev. Geophys., 31, 357-396, doi: 10. 1029/93RG02030. [Link]

Savage, J. C., W. Gan, and J. L. Svarc, 2001: Strain accumulation and rotation in the Eastern California Shear Zone. J. Geophys. Res., 106, B10, 21995-22007, doi: 10.1029/2000JB000127. [Link]

Savage, J. C., W. Gan, W. H. Prescott, and J. L. Svarc, 2004: Strain accumulation across the Coast Ranges at the latitude of San Francisco, 1994-2000. J. Geophys. Res., 109, B03413, doi: 10.1029/2003JB002612. [Link]

Schaffrin, B., 2006: A note on Constrained Total LeastSquares estimation. Linear Alg. Appl., 417, 245-258, doi: 10.1016/j.laa.2006.03.044. [Link]

Schaffrin, B. and A. Wieser, 2008: On weighted total leastsquares adjustment for linear regression. J. Geodesy, 82, 415-421, doi: 10.1007/s00190-007-0190-9. [Link]

Segall, P. and J. L. Davis, 1997: GPS applications for geodynamics and earthquake studies. Annu. Rev. Earth Planet. Sci., 25, 301-336, doi: 10.1146/annurev.earth. 25.1.301. [Link]

Shen, Z. K., J. Lü, M. Wang, and R. Bürgmann, 2005: Contemporary crustal deformation around the southeast borderland of the Tibetan Plateau. J. Geophys. Res., 110, B11409, doi: 10.1029/2004JB003421. [Link]

Tang, R., W. Han, Z. Huang, H. Zian, and Y. Zhang, 1993:
Active Faults and Earthquakes in Sichuan Province. Seismol. Publ. House, Beijing, 360 pp.

van Huffel S. and J. Vandewalle, 1991: The Total Least Squares Problem: Computational Aspects and Analysis, SIAM, Philadelphia, 42 pp.

Vergnolle, M., E. Calais, and L. Dong, 2007: Dynamics of continental deformation in Asia. J. Geophys. Res., 112, B11403, doi: 10.1029/2006JB004807. [Link]

Wang, E., B. C. Burchfiel, L. H. Royden, L. Chen, J. Chen, W. Li, and Z. Chen, 1998: Late Cenozoic Xianshuihe-Xiaojiang, Red River, and Dali Fault Systems of Southwestern Sichuan and Central Yunnan, China. Geological Society of Amer, 327, 108 pp.

Wang, L.Y., C. J. Xu, and T. D. Lu, 2010: Inversion of strain parameter using distance changes based on total least squares. Geomatics and Information Science of Wuhan University, 35, 181-184.

Wang, Y. Z., E. Wang, Z. K. Shen, M. Wang, W. J. Gan, X. J. Qiao, G. J. Meng, T. M. Li, W. Tao, Y. L. Yang, J. Chen, and P. Li, 2008: Present-day motion velocity of main fault inversion based on GPS data restriction in Sichuan-Yunnan region. Sci. China Ser. D: Earth Sci., 38, 582-597.

$\mathrm{Xu}, \mathrm{C}$. and Y. Wen, 2007: Identification and analysis of crustal motion and deformation models in the SichuanYunnan region. J. Appl. Geodesy, 1, 213-222, doi: 10. 1515/jag.2007.023. [Link]

Xu, C., W. B. Shen, and D. B. Chao, 2006: Geophysical Geodesy Principles and Methods. Wuhan University Press, 193 pp.

Xu, X., X. Wen, R. Zheng, W. Ma, F. Song, and G. Yu, 2003: Pattern of latest tectonic motion and its dynamics for active blocks in Sichuan-Yunnan region, China. Sci. China Ser. D: Earth Sci., 46, 210-216, doi: 10.1360/03dz0017. [Link]

Zhang, C., F. Xie, and S. Zhang, 2001: A modeling study on the controlling factors of seismic source distribution and their strength index - Practical analyses of the seismic environmental factors of the Honghe fault. Acta Seismol. Sin., 14, 129-140, doi: 10.1007/s11589-001$0143-\mathrm{y}$. [Link]

Zhou, S. J. and T. D. Lu, 2008: The Computation for Linear Regression of Bi-variable. Jiangxi Sci., 26, 109-111. 